\title{
Effects of the turbulent ISM on radio observations of quasars
}

\section{Krisztina Éva Gabányi*}

Max-Planck-Institut für Radioastronomie, Bonn, Germany

E-mail: gabanyikempifr-bonn.mpg.de

S. Britzen, T. P. Krichbaum, U. Bach, L. Fuhrmann, A. Kraus, A. Witzel and J. A. Zensus

Max-Planck-Institut für Radioastronomie, Bonn, Germany

E-mail: sbritzen@mpifr-bonn.mpg.de, tkrichbaumempifr-bonn.mpg.de

ubachempifr-bonn.mpg . de fuhrmannempifr-bonn.mpg . de.

akrausempifr-bonn.mpg.de awitzelempifr-bonn.mpg.de

azensusempifr-bonn.mpg.de

In radio bands, the study of compact radio sources can be affected by propagation effects introduced by the interstellar medium, usually attributed to the presence of turbulent intervening plasma along the line of sight. Here, two of such effects are presented. The line of sight of B 2005+403 passes through the heavily scattered region of Cygnus causing substantial angular broadening of the source images obtained at frequencies between $0.6 \mathrm{GHz}$ and $8 \mathrm{GHz}$. At higher frequencies, however, the intrinsic source structure shines through. Therefore, multi-frequency VLBI observations allow to study the characteristics of the intervening material, the source morphology and the interplay between them in forming the observed image.

(This article is based upon the published paper of Gabányi et al. For more details see [6].)

8th European VLBI Network Symposium

September 26-29, 2006

Toruń, Poland

\footnotetext{
* Speaker.
} 


\section{Introduction}

The propagation of radio waves through the ionized interstellar medium (ISM) causes several effects, such as Faraday rotation and depolarization of polarized emission, dispersion of pulsar signals, scatter broadening of compact radio sources and intensity fluctuations caused by diffractive and refractive interstellar scintillation (ISS). Detailed study of scatter broadening in compact radio sources can lead to a better understanding of the interstellar medium.

Another prominent propagation effect addressed here is the so-called Intraday Variability (IDV, [8]), and the question of how it is related to the ISS. IDV surveys show that a large fraction (up to $30 \%$ ) of all compact flat-spectrum radio sources show this effect, characterized by variations of up to $20-30 \%$ and variability timescales ranging from less than one hour to several days (e.g. [10], [12] and references therein). If interpreted as resulting from intrinsic incoherent emission processes in the source, such short variability timescales imply - via the light travel time argument - apparent brightness temperatures of $T_{\mathrm{B}}=10^{16} \mathrm{~K}-10^{19} \mathrm{~K}$, far in excess of the inverse-Compton limit ([9]). With the assumption of relativistic Doppler-boosting, the brightness temperatures can be reduced; this, however, would require uncomfortably large Doppler boosting factors of $\delta \simeq 20-200$.

Alternatively, IDV in the radio-bands could also be explained extrinsically as a result of scattering of radio waves in the turbulent ISM of our Galaxy (e.g. [15]). The main problem of this interpretation is that it cannot explain the radio-optical broad-band correlations observed in at least some sources ([14]). Recent detection of diffractive ISS in J 1819+3845 implies micro-arcsecond source sizes and brightness temperatures of $\sim 10^{14} \mathrm{~K}$, which again requires Doppler boosting factors of $\delta \simeq 100$ ([13]). It is therefore likely that the IDV phenomenon involves both, a combination of intrinsic and extrinsic effects (e.g. [11]).

We present measurements of the scatter-broadened quasar B 2005+403, that combined VLBI and flux density variability measurements obtained at various frequencies. The scatter broadening observed in the VLBI images at lower frequencies has been measured and yields further constraints to the properties of the intervening ISM. Additional parameters for the ISM are obtained from the IDV monitoring of the source carried out with the Effelsberg 100-m telescope.

B 2005+403 is a flat-spectrum quasar $\left(\alpha_{0.3 / 5 \mathrm{GHz}}=0.3, S \sim v^{\alpha}\right.$; [1] $)$ at a redshift of $z=1.736$ ([2]). It is located close to the Galactic plane $\left(l=76.82^{\circ}, b=4.29^{\circ}\right)$ behind the Cygnus superbubble region. Earlier studies showed that interstellar scattering affects the VLBI image of the source, causing angular broadening at frequencies below $5 \mathrm{GHz}$ (see [4] and references therein). The high value of the scattering measure $\left(\mathrm{SM}=\int_{0}^{L} C_{N}^{2}(s) d s^{1}\right.$ ), derived for the line of sight of B $2005+403$ by [5], reflects the strong influence of the interstellar medium.

\section{VLBI data and data reduction}

The VLBI observations of B $2005+403$ were made at frequencies between $1.6 \mathrm{GHz}$ and $43 \mathrm{GHz}$. After correlation with the VLBI correlator in Socorro (NRAO) or Bonn (MPIfR), a priori amplitude calibration using system temperature measurement and fringe-fitting were carried out

\footnotetext{
${ }^{1}$ The Scattering Measure is the path integral over the coefficient $C_{N}^{2}$ of the electron density fluctuation wavenumber spectrum.
} 


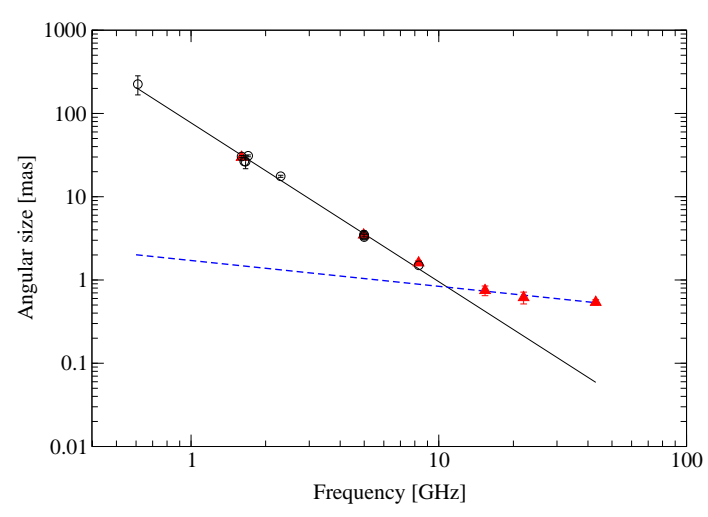

Figure 1: The measured angular size plotted versus observing frequency. The solid black line represents a power-law fit to the data in the range from $0.6 \mathrm{GHz}$ to $8 \mathrm{GHz}$. The dashed blue line represents a power law fitted to the data in the range from $15 \mathrm{GHz}$ to $43 \mathrm{GHz}$. Black circles denote data from the literature (沛], 沛), red triangles denote our data.

\begin{tabular}{|c|c|c|c|}
\hline $\begin{array}{c}v \\
{[\mathrm{GHz}]}\end{array}$ & Epoch & $\begin{array}{c}\theta \\
{[\mathrm{mas}]}\end{array}$ & Ref. \\
\hline 0.6 & Oct 1986 & $225.0 \pm 58.0$ & {$[5]$} \\
\hline 1.6 & 1998.14 & $29.8 \pm 0.5$ & [6] \\
\hline 1.7 & Mar 1986 & $26.4 \pm 4.7$ & [5] \\
\hline 1.7 & Jan 1997 & $31.0 \pm 0.8$ & [4] \\
\hline 2.3 & Jan 1997 & $17.6 \pm 0.5$ & 所 \\
\hline 5 & Oct 1985 & $3.5 \pm 0.4$ & [5] \\
\hline 5 & 1996.82 & $3.5 \pm 0.1$ & [6] \\
\hline 5 & Jan 1997 & $3.4 \pm 0.1$ & 《4 \\
\hline 8 & 1996.83 & $1.6 \pm 0.1$ & [6] \\
\hline 15 & 1996.73 & $0.8 \pm 0.1$ & {$[\sqrt{6}]$} \\
\hline 22 & 1996.73 & $0.6 \pm 0.1$ & [6] \\
\hline 43 & 1996.73 & $0.5 \pm 0.1$ & {$[6]$} \\
\hline
\end{tabular}

Table 1: The measured angular sizes of B 2005+403 at different frequencies.

with the standard AIPS procedures. Editing, phase and amplitude self-calibration and imaging were carried out using AIPS and the Caltech Difmap packages.

To study the scattering effects following [河], we measured the total angular source size as a function of observing frequency by fitting the visibility data of B $2005+403$ at all frequencies with one Gaussian component within Difmap.

Additional angular-size measurements of B 2005+403 were taken from [5] and [ 4 ].

\section{Propagation effect I: Scatter broadening of B 2005+403}

The angular size of B 2005+403 versus frequency is plotted in Fig. 1, using our data and earlier measurements from the literature. The corresponding values are given in Table 11. To quantify the scatter broadening, which dominates at lower frequencies, a power law was fitted to the sizefrequency relation in the frequency range from $0.67 \mathrm{GHz}$ to $8 \mathrm{GHz}$, yielding $\theta=(77.1 \pm 4.0)$. $(v / 1 \mathrm{GHz})^{-(1.91 \pm 0.05)}$ mas. This fit is shown as black line in Figure 1. (Exclusion of the 8-GHz data point does not change the slope significantly, yielding: $\theta=(77.7 \pm 4.0) \cdot(v / 1 \mathrm{GHz})^{-1.92 \pm 0.06}$.)

Above $8 \mathrm{GHz}$, the extrapolated scattering size becomes smaller than the measured source size. This indicates that towards higher frequencies scattering effects are less dominant and that the intrinsic structure of the sources shines through. The differences between the extrapolated scattering size and the measured source size at all three frequencies $(15 \mathrm{GHz}, 22 \mathrm{GHz}$, and $43 \mathrm{GHz})$ are of the order of 0.4 mas.

To characterize the dependence of the intrinsic source size on frequency, a power law was fitted to the size-frequency relation above $8 \mathrm{GHz}$, yielding: $\theta_{\text {int }}=(1.7 \pm 0.4) \cdot(v / 1 \mathrm{GHz})^{-(0.31 \pm 0.07)}$ mas (dashed blue line in Fig. 1). With this fit, it was possible to obtain an upper limit on the intrinsic source size at lower frequencies where direct size measurements with VLBI are not possible. 


\begin{tabular}{lccccc}
\hline \hline Source name & $\langle S\rangle[\mathrm{Jy}]$ & $\sigma[\mathrm{Jy}]$ & $m[\%]$ & $Y[\%]$ & $\chi_{\mathrm{r}}^{2}$ \\
\hline \multicolumn{1}{l}{$v=1.6 \mathrm{GHz}, m_{0}=0.25 \%$} \\
\hline B 2005+403 & 2.430 & 0.024 & 1.01 & 2.93 & 5.593 \\
NGC 7027 & 1.906 & 0.004 & 0.19 & 0 & 0.197 \\
B 2021+614 & 2.193 & 0.006 & 0.25 & 0 & 0.365 \\
\hline \hline \multicolumn{7}{l}{$v=5 \mathrm{GHz}, m_{0}=0.20 \%$} \\
\hline B 2005+403 & 2.905 & 0.013 & 0.45 & 1.18 & 3.107 \\
NGC 7027 & 5.489 & 0.011 & 0.20 & 0 & 0.621 \\
\hline
\end{tabular}

Table 2: The variability parameters of B $2005+403$ and the secondary calibrators at $1.6 \mathrm{GHz}$ (top) and $5 \mathrm{GHz}$ (bottom). Col. 2 shows the mean flux density in Jy, col. 3 its standard deviation, col. 4 the modulation index, col. 5 the noise-bias corrected variability amplitude, and col. 6 the reduced $\chi_{\mathrm{r}}^{2}$. For a detailed definition of these values, see [10].

Hence, a lower limit on the brightness temperature could also be calculated. Below $8 \mathrm{GHz}$, the intrinsic source sizes are in the range of $1-1.5$ mas. The corresponding lower limits to the brightness temperatures at $1.6 \mathrm{GHz}, 5 \mathrm{GHz}$ and $8 \mathrm{GHz}$ are $T_{\mathrm{B}} \geq(0.6 \pm 0.5) \cdot 10^{12} \mathrm{~K}, T_{\mathrm{B}} \geq(0.14 \pm 0.09) \cdot 10^{12} \mathrm{~K}$, and $T_{\mathrm{B}} \geq(0.08 \pm 0.04) \cdot 10^{12} \mathrm{~K}$, respectively. These numbers are in accordance with typical brightness temperatures measured with VLBI and neither strongly violate the inverse-Compton limit nor do they indicate excessive Doppler-boosting.

The size of the scattering disk at a given frequency can be estimated from the deconvolution formula: $\theta_{\text {scat }}=\sqrt{\theta_{\text {obs }}^{2}-\theta_{\text {int }}^{2}}$. From the power-law fit, the scattering size at $1 \mathrm{GHz} \theta_{1 \mathrm{GHz}}=$ $(77.1 \pm 4.0)$ mas. Following [16] and assuming a Kolmogorov turbulence, the scattering measure can be calculated. The derived SM of $(0.43 \pm 0.04) \mathrm{m}^{-20 / 3} \mathrm{kpc}$ is consistent with the previous measurement of [5], though with much improved accuracy.

\section{Propagation effect II: IDV behaviour of B 2005+403}

In November and December 2003, the flux-density variability of B 2005+403 was monitored with high time resolution using the Effelsberg 100-m radio telescope. In Table 2, the results of these measurements are summarized. To characterize the variability amplitudes and their significance, we follow the methods described in [10]. Column 2 of the table gives the average flux density, col. 3 the standard deviation, col. 4 the modulation index $(m=100 \cdot \sigma /\langle S\rangle)$, col. 5 the variability amplitude (defined as $Y=3 \sqrt{m^{2}-m_{0}^{2}}$, where $m_{0}$ is the modulation index of a source assumed to be stationary during the observation), and col. 6 the reduced $\chi^{2}$. The value of $Y$ is only given, if the $\chi^{2}$-test gives a higher than $99.9 \%$ probability for significant variations.

The variability amplitude of B 2005+403 decreases with frequency. Formally, we measure a modulation index of $m=1.01 \%$ at $1.67 \mathrm{GHz}$ and of $m=0.45 \%$ at $5 \mathrm{GHz}$. At this frequency, the detection of IDV is marginal. We note that the model of refractive ISS in the weak regime predicts a decrease of the modulation index with increasing frequency ([15]). This is consistent with our observations.

The source size and the apparent brightness temperature can be determined from the interstellar scintillation model, assuming that the main reason for the observed variability is the motion 
of the Earth through the scintillation pattern. In this scenario, the variability time-scale in days is ([15]): $t=18 \cdot \theta_{\mathrm{scat}} \cdot L_{\mathrm{kpc}} \cdot\left(\frac{v}{50 \mathrm{~km} \mathrm{~s}^{-1}}\right)^{-1}$.

Cordes and Lazio modeled the electron density turbulences of the Milky Way (NE2001, [3]). They assumed a distance of $2.35 \mathrm{kpc}$ to the scattering "clump" (or screen) responsible for the angular broadening of B $2005+403$. The characteristic variability time-scales derived from the light curves is less than a day for B $2005+403$. With a scattering size of $\theta_{\text {scat }}=30$ mas at $1.6 \mathrm{GHz}$, the screen distance of $2.35 \mathrm{kpc}$ and typical Galactic velocities of $\leq 220 \mathrm{~km} / \mathrm{s}$, one would expect to see variations on time-scales $\geq 288$ days. Thus, a screen located at kpc distance cannot explain the observed short variability time-scale.

To reproduce a variability time-scale of $\leq 1$ day at $1.6 \mathrm{GHz}$ within the aforementioned model, the following constraint for the product of the scattering size and the screen distance is obtained: $\theta_{\mathrm{eff}} L \leq 2.2 \cdot 10^{-2}$ mas kpc. For the measured scattering size of $\sim 30$ mas, this leads to an unreasonably nearby screen of $\leq 1 \mathrm{pc}$. The only way out of this dilemma is a smaller scattering size. Adopting a minimum screen distance of at least $10 \mathrm{pc}$, as required for the interpretation of the ultra fast scintillators ([13] and references therein), one obtains $\theta_{\text {scat }} \leq 2.2$ mas.

An upper limit on the distance of the screen is obtained from the restriction of the source size via the inverse-Compton limit of $T_{\mathrm{B}}^{\mathrm{IC}}=10^{12} \mathrm{~K}$. The requirement that the brightness temperature be lower than this, leads to a source size of $\theta_{\text {int }}=\sqrt{1.77 \cdot 10^{12} S_{v} v^{-2} T_{\mathrm{B}}^{-1} \delta^{-1}(1+z)} \geq 0.6$ mas, where $S_{1.6 \mathrm{GHz}}=2.4 \mathrm{Jy}$ is adopted from Table 2 , the Doppler factor $\delta \simeq 10$, and $v$ is in the units of GHz. Approximating $\theta_{\text {scat }}$ with this size and putting a relative screen velocity in the range from $50 \mathrm{~km} / \mathrm{s}$ to $220 \mathrm{~km} / \mathrm{s}$, leads to an upper limit of the screen distance in the range from $9 \mathrm{pc}$ to $41 \mathrm{pc}$.

\section{Conclusion}

The IDV observed in B 2005+403 can be explained with a scattering screen located at a distance less than or equal to $41 \mathrm{pc}$ that can be characterized by a scattering size of 0.6 mas $\leq \theta_{\text {scat }} \leq$ 2.2 mas. The corresponding scattering measure at $1.6 \mathrm{GHz}$ is then of the order of $10^{-3} \mathrm{~m}^{-20 / 3} \mathrm{kpc}$. These values are considerably lower than the scattering measure of the more distant screen that is thought to be responsible for the scatter broadening in B 2005+403. Therefore, the observed IDV cannot be caused by that distant screen. Most likely, one has to assume multiple scattering by at least two spatially and physically very different plasma screens. In this scenario, the first screen contributes to a significant scatter broadening of the source image, whereas the second screen accounts for only very weak scattering due to large quenching effects $\left(\theta_{\text {source }}>>\theta_{\text {scat }}\right)$. The quenched scattering ([15]) by the second screen can explain the relatively low variability amplitudes of $\leq 1 \%$, observed in the IDV experiments at $5 \mathrm{GHz}$ and $1.6 \mathrm{GHz}$. Quantitatively, this can be verified using equation (20) of [7], which relates the variability index, the scattering measure, the effective source size, and the screen distance. With the parameters as above, a modulation index of $m \leq 1 \%$ is obtained, in good agreement with the observations.

\section{References}

[1] R. H. Becker, R. L. White, A. L. Edwards, A new catalog of 53,522 $4.85 \mathrm{GHz}$ sources, ApJS 75 (1991). 
[2] A. Boksenberg, S. A. Briggs, R. F. Carswell, 2005+403 - A QSO near the galactic plane, MNRAS 177 (1976).

[3] J. M. Cordes, T. J. W. Lazio, NE2001. I. A New Model for the Galactic Distribution of Free Electrons and its Fluctuations, preprint, astro-ph/0207156 (2002).

[4] K. M. Desai, A. L. Fey, Anisotropic Interstellar Scattering toward the Cygnus Region, ApJS 133 (2001).

[5] A. L. Fey, S. R. Spangler, R. L. Mutel, VLBI angular broadening measurements in the Cygnus region, ApJ 337 (1989).

[6] K. É. Gabányi, T. P. Krichbaum, S. Britzen, et al., High frequency VLBI observations of the scatter-broadened quasar B2005+403, A\&A 451 (2006)

[7] J. Goodman, Radio scintillation of gamma-ray-burst afterglows, New Astronomy 2 (1997).

[8] D. S. Heeschen, T. P. Krichbaum, C. J. Schalinski, et al., Rapid variability of extragalactic radio sources, AJ 94 (1987).

[9] K. I. Kellermann, I. I. K. Pauliny-Toth, The Spectra of Opaque Radio Sources, ApJ 155 (1969).

[10] A. Kraus, T. P. Krichbaum, R. Wegner, Intraday variability in compact extragalactic radio sources, $A \& A 401$ (2003).

[11] T. P. Krichbaum, A. Kraus, L. Fuhrmann, et al., Intraday Variability in Northern Hemisphere Radio Sources, PASA 19 (2002).

[12] J. E. J. Lovell, D. L. Jauncey, H. E. Bignall, First Results from MASIV: The Microarcsecond Scintillation-induced Variability Survey, AJ 126 (2003).

[13] J.-P. Macquart, A. G. de Bruyn, Diffractive Interstellar Scintillation of the Quasar J1819+3845 at $21 \mathrm{~cm}, A \& A 446$ (2006).

[14] A. Quirrenbach, A. Witzel, S. Wagner, et al., Correlated radio and optical variability in the BL Lacertae object 0716+714, ApJ 372 (1991).

[15] B. J. Rickett, T. J. W. Lazio, F. D. Ghigo, Interstellar Scintillation Observations of 146 Extragalactic Radio Sources, ApJS 165 (2005).

[16] J. H. Taylor, J. M. Cordes, Pulsar distances and the galactic distribution of free electrons, ApJ 411 (1993). 